\title{
Continuous Glucose Monitors and Tight Glycaemic Control in Intensive Care: An In-Silico Proof of Concept Analysis
}

\author{
Matthew SIGNAL*, Christopher G. PRETTY*, Aaron LE COMPTE*, \\ Geoffrey M. SHAW**, J. Geoffrey CHASE* \\ *Centre for Bioengineering, University of Canterbury, New Zealand \\ ** Department of Intensive Care Medicine, Christchurch Hospital, New Zealand
}

\begin{abstract}
Tight glycaemic control (TGC) in critical care has shown distinct benefits, but has also proven difficult to obtain. The risk of severe hypoglycaemia $(<2.2 \mathrm{mmol} / \mathrm{L})$ raises significant concerns for safety. Continuous Glucose Monitors (CGMs) offer frequent, though potentially noisy, automated measurement and thus the possibility of using them for early detection and intervention of hypoglycaemic events. This in-silico study investigates the potential of CGM devices to maintain control, prevent hypoglycaemia and reduce clinical effort. Retrospective clinical data from the SPRINT TGC study covering 26 patients was used with clinically validated metabolic system models and 3 different stochastic noise models (two Gaussian and one first-order autoregressive.) The noisy, virtual CGM blood glucose (BG) values were filtered and used to drive the SPRINT TGC protocol. A simple threshold alarm was used to trigger glucose interventions to avert potential hypoglycaemia. Monte Carlo analysis was used to get robust results from the stochastic noise models. Using SPRINT with simulated CGM noise, the BG time in the 4.4-6.1 mmol/L band was reduced no more than $3 \%$ from $45.2 \%$ obtained with glucometer sensors. The number of patients experiencing severe hypoglycaemia was reduced by $0-30 \%$. Duration of hypoglycaemic events was reduced by $19-65 \%$. Finally, nurse workload was reduced by approximately 20 minutes per patient, per day. The results of this proof of concept study justify a pilot clinical study for verification in a clinical setting.
\end{abstract}

Keywords: Hypoglycaemia, CGM, continuous glucose monitor, alarm, glycaemic control, blood glucose, sensor.

\section{INTRODUCTION}

Critically ill patients often experience high levels of insulin resistance and stress-induced hyperglycaemia, (Finney et al., 2003, Krinsley, 2004) which can negatively impact outcomes (Capes et al., 2000, Finney et al., 2003). Some studies have shown that tight glycaemic control (TGC) can reduce intensive care unit (ICU) patient mortality by up to $45 \%$ (Van den Berghe et al., 2001, Krinsley, 2004, Chase et al., 2008c). Although there is little agreement on what constitutes desirable glycaemic performance (Mackenzie et al., 2005), the primary aim of TGC is to reduce elevated blood glucose with minimal hypoglycaemia. Two major causes of hypoglycaemia are reported to be clinical error and/or infrequent BG measurement using bedside glucometers or blood gas analysers (Chase et al., 2008a, Braithwaite et al., 2006).

Typically, in most ICU studies blood glucose is measured 1-4 hourly and more frequently only if the levels are already at or near hypoglycaemia. More frequent measurement (even 1-2 hourly) is uncommon due to the clinical effort required. The result can be extremely variable glycaemic control with longer measurement intervals. Thus, continuous glucose monitors (CGMs) with their rapid 2-10 minute measurement rates may allow more tightly controlled BG levels.
There have been relatively few successful investigations of CGMs in critical care use (Goldberg et al., 2004). Added sensor noise is a trade off (in some cases) of the CGMs far higher, automated sampling rate (Goldberg et al., 2004, Clarke et al., 2005) and excess sensor noise must be effectively managed. However, these sensor and algorithm technologies are also constantly evolving, with every new generation offering improvements (Klonoff, 2005).

This study examines how ICU patients might behave on the SPRINT TGC protocol driven by simulated CGM measurements rather than the normal 1-2 hourly glucometer measurements. Hypoglycaemia detection and prevention are also tested, as CGMs offer the ability to detect and avert these events. This in-silico study thus aims to demonstrate that CGM devices coupled with an effective TGC protocol are capable of reducing nursing workload, and increasing safety, while maintaining TGC.

\section{SUBJECTS AND METHODS}

\subsection{Subjects:}

This study uses retrospective clinical data from 26 patients, comprising of the 20 patient benchmark cohort from the SPRINT TCG study (Chase et al., 2008b) and 6 additional SPRINT patients who experienced at least one genuine hypoglycaemic event during their stay. SPRINT is a TGC 
protocol implemented in Christchurch ICU since August 2005 that modulates both insulin and dextrose nutrition. BG data is sampled 1-2 hourly using bedside devices via either arterial line or fingerstick. Each patient spent 5 days or longer on SPRINT, during which time some experienced breaks in treatment, typically due to surgery or other clinical changes. Patients who experienced breaks from SPRINT had their BG data segmented into 2 or more un-interrupted episodes (at least 12 hours), resulting in 55 individual continuous data sets. Each episode was then treated as an individual 'patient' for the purposes of this study. Details of the patient cohort are shown in Table 1. The use of these patient records falls under existing ethics approval granted by the Upper South Regional Ethics Committee, New Zealand.

Table 1. Cohort details, presented as median [interquartile range] where applicable.

\begin{tabular}{|c|c|}
\hline Number of Patients, $\mathrm{N}$ & 55 \\
\hline Age & $\begin{array}{c}66 \\
{[59-73]}\end{array}$ \\
\hline Gender & $65 \%$ Male \\
\hline Length of SPRINT (hours) & $\begin{array}{c}139 \\
{[42-210]}\end{array}$ \\
\hline Number of clinical Measurements & $\begin{array}{c}77 \\
{[27-146]}\end{array}$ \\
\hline APACHE II Score & $\begin{array}{c}20 \\
{[15-27]}\end{array}$ \\
\hline APACHE II risk of death (\%) & $\begin{array}{c}33.6 \\
{[13.7-56.1]}\end{array}$ \\
\hline
\end{tabular}

\subsection{Methods - Insulin-Glucose Regulatory System Model:}

This study uses a model derived from the clinically validated glucose-insulin model reported in (Lin et al., 2008a). Modifications to the model include removal of the basal set point term due to the difficulty of obtaining it for critical care patients, and, addition of endogenous glucose production (EGP) and central nervous system (CNS) uptake. Finally, a 2 compartmental feed model was used to ensure mass conservation of ingested glucose.

\subsection{Methods-CGM Noise Models:}

This study uses two different types of stochastic noise model. One simple, Gaussian model based on data from an ICU trial of CGM sensors (Goldberg et al., 2004), and one first-order autoregressive model based on an analysis of data from individuals with ambulatory type 1 diabetes (Breton and Kovatchev, 2008).

Two levels of Gaussian noise model are investigated. The first is designed to produce equivalent simulated CGM BG measurement errors on a similar cohort to those reported in (Goldberg et al., 2004) in a 2004 study of the Medtronic CGMS (Minimed-Medtronic, Northridge, CA) device in a medical ICU. The second Gaussian model had the magnitude of additive noise reduced by half to simulate improvements in technology since 2004. Both models used independent, identically distributed Gaussian based noise as described in (Pretty et al., 2010).

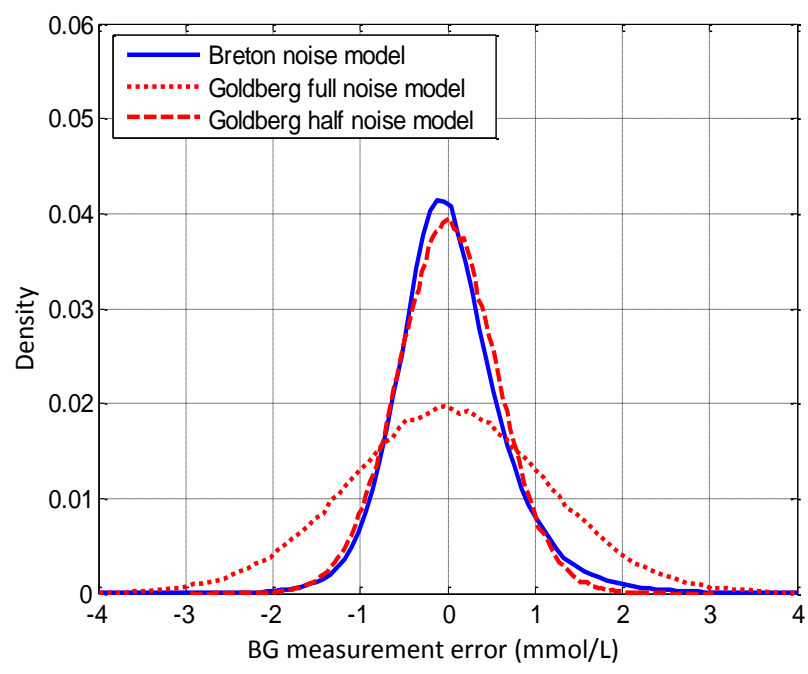

Figure 1. Error distributions of noise models used in this study.

The autoregressive noise model implemented in this paper is that described in (Breton and Kovatchev, 2008) in their 2008 study of CGM error in ambulatory type 1 diabetics. This model is based on data from the FreeStyle Navigator CGM sensor (Abbott Diabetes Care, Alameda, CA). The model consists of two parts, a delay modelling the diffusion time from plasma to interstitium and sensor lag, and non-Gaussian sensor noise. The sensor noise is modelled as first-order autoregressive with the random component taken from an unbounded Johnson system as reported in (Breton and Kovatchev, 2008).

Figure 1 compares the error distributions obtained on this cohort for each of the error models used in this study. The distributions for the Breton and Goldberg-half magnitude models are very similar despite their time-series behaviour being quite different. However, the similarity does show a measure of technological advancement since the original Goldberg study (Goldberg et al., 2004).

Calibration drift due to sensor degradation over time was not considered in this study, as this factor is controlled by the specific calibration protocol used with the sensor and has not been reported in detail to the authors knowledge.

\subsection{Methods - In-Silico CGM Measurements:}

Model generated blood glucose profiles for each patient were sampled incrementally at 5 minute intervals. As these 'actual' BG measurements were sampled in-silico, noise was added from a noise model, creating incremental virtual CGM measurement sequences.

\subsection{Methods - CGM Filtering:}

The virtual CGM sensor BG measurements and error were implemented using MATLAB ${ }^{\mathrm{TM}}$ (The Mathworks; Natick, MA). The noisy measurements were filtered incrementally, simulating the process that would be encountered in a real- 
time clinical setting. A median filter as described in (Pretty et al., 2009) was used in this study.

\subsection{Methods - Hypoglycaemia Alarm/Intervention Design:}

This study used a simple threshold algorithm to trigger an alarm and glucose intervention when a hypoglycaemic event appeared imminent. The criteria for triggering an alarm and intervention were:

- Two consecutive CGM measurements $<3 \mathrm{mmol} / \mathrm{L}$

- A negative BG gradient.

- At least 15 minutes since the last intervention

Two different sized glucose interventions were used in this study. A 12.5 gram intra-venous (IV) bolus was given over 3 minutes, unless a SPRINT BG measurement (1-2 hourly) had been taken in the previous 15 minutes. If a SPRINT measurement had been taken, the size of the bolus was reduced to 6 grams, to reduce interference with any increase in nutritional prescribed by the protocol. The 15 minute period between consecutive interventions replicates typical clinical practice, allowing time to see a significant outcome BG change.

\subsection{Methods - Simulating SPRINT with CGM Measurements:}

A single filtered measurement was taken 1-2 hourly from the full sequence of filtered CGM data and used to drive the SPRINT protocol, with no other modifications to the algorithm. Thus, using CGM with all else equal would save significant nursing time by removing the need for a finger stick measurement every 1-2 hours (mean 16/day) (Chase et al., 2008c). While the SPRINT protocol only used 1-2 hourly values from CGM sequence, the full set of data was used for the hypoglycaemia detection and prevention part of the study.

2.7 Methods - Analysis:

Monte Carlo simulations were performed using the three stochastic noise models, with 10 runs for each of the $55 \mathrm{BG}$ episodes. The baseline simulation (no added noise) was performed once, for comparison.

Time in the 4.4-6.1 mmol/L BG band and time spent in hypoglycaemia (below $2.2 \mathrm{mmol} / \mathrm{L}$ ) were considered the most important metrics for this investigation. These metrics were calculated using two different methods. The first method used only the 1-2 hourly filtered CGM measurements driving SPRINT, allowing a fair comparison with the clinical data. The second method used the full, continuous CGM data sequence to simulate what might be seen in a clinical situation if CGMs were used.

Nutrition and insulin interventions prescribed by SPRINT were also examined. Differences in these interventions compared to the baseline SPRINT results were analysed to determine the impact of CGM sensor noise on operation of this protocol.

Results are presented as non-parametric (median [interquartile range]) or lognormal (mean [1 standard deviation range]) as indicated. Lognormal statistics reflect the skewed (non-Gaussian) results obtained for BG levels.

\section{RESULTS}

Tables 2, 3, 4 and 5 show the level of control that was achieved when each of the noise models was implemented. Results in Tables 2, 3 and 4 were determined using only the 1-2 hourly SPRINT BG measurements, allowing a fair comparison with the clinical baseline results. Table 5 shows

Table 2. Overall cohort summary statistics as reported by 1-2 hourly filtered CGM from Monte Carlo simulations using each of the noise models (with alarm and 12.5 gram interventions).

\begin{tabular}{|l|c|c|c|c|}
\hline Summary Statistics & Clinical Baseline & Breton & Goldberg (full) & Goldberg (half) \\
\hline Num 1-2 hourly BG measurements [IQR]: & 5816 & $5923[5897-5948]$ & $5978[5974-5988]$ & $5822[5811-5855]$ \\
\hline BG median [IQR] (mmol/L): & 5.6 & $5.6[4.7-6.6]$ & $5.7[4.7-6.6]$ & $5.6[4.7-6.5]$ \\
\hline$\%$ BG within 4.4-6.1 mmol/L [IQR] & 45.2 & $43.4[43.3-43.8]$ & $42.4[42.2-42.6]$ & $46.1[45.2-46.3]$ \\
\hline Median insulin rate [IQR] (U/hr): & $3.0[2.0-3.0]$ & $3.0[1.0-3.0]$ & $3.0[1.0-3.0]$ & $3.0[2.0-3.0]$ \\
\hline Median glucose rate [IQR] (g/hr): & $5.2[3.3-6.3]$ & $5.2[3.3-6.5]$ & $5.2[3.3-6.5]$ & $5.2[3.3-6.5]$ \\
\hline
\end{tabular}

Table 3. Overall cohort hypoglycaemia/intervention statistics as reported by 1-2 hourly filtered CGM from Monte Carlo simulations using each of the noise models (with alarm and 12.5 gram interventions).

\begin{tabular}{|l|c|c|c|c|}
\hline Hypoglycaemia/Intervention statistics & Clinical Baseline & Breton & Goldberg (full) & Goldberg (half) \\
\hline$\% \mathrm{BG}<4.4 \mathrm{mmol} / \mathrm{L}[\mathrm{IQR}]$ & 19.7 & $19.7[19.4-19.8]$ & $20.7[20.5-20.7]$ & $19.8[19.7-19.9]$ \\
\hline$\% \mathrm{BG}<2.2 \mathrm{mmol} / \mathrm{L}[\mathrm{IQR}]$ & 0.4 & $0.5[0.5-0.6]$ & $0.3[0.3-0.4]$ & $0.2[0.2-0.3]$ \\
\hline Num patients $<2.2 \mathrm{mmol} / \mathrm{L}[\mathrm{IQR}]$ & 12.0 & $12[11-12]$ & $10[8-11]$ & $8[6-9]$ \\
\hline Number of interventions (where applicable) & 0 & 356 & 330 & 244 \\
\hline
\end{tabular}

Table 4. Per-patient summary statistics as reported by 1-2 hourly filtered CGM from Monte Carlo simulations using each of the noise models (with alarm and 12.5 gram interventions).

\begin{tabular}{|l|c|c|c|c|}
\hline Per-patient statistics & Clinical Baseline & Breton & Goldberg (full) & Goldberg (half) \\
\hline Hours of control [IQR]: & $137.0[41.3-208.3]$ & $138.0[41.3-208.3]$ & $137.0[41.0-208.5]$ & $137.0[41.3-208.3]$ \\
\hline Num 1-2 hourly BG measurements [IQR]: & $79.0[36.8-119.0]$ & $82.0[36.3-121.8]$ & $82.0[32.3-122.0]$ & $82.0[36.3-118.8]$ \\
\hline BG median [IQR] (mmol/L) & $5.8[4.8-6.2]$ & $5.8[4.9-6.2]$ & $5.8[4.8-6.2]$ & $5.8[4.7-6.2]$ \\
\hline$\%$ BG within 4.4-6.1 mmol/L [IQR] & 41.7 & $40.0[41.1-41.9]$ & $39.0[38.5-39.6]$ & $42.3[41.6-43.0]$ \\
\hline Median insulin rate [IQR] (U/hr): & $3.0[1.2-3.0]$ & $3.0[2.0-3.0]$ & $3.0[1.2-3.0]$ & $3.0[1.2-3.0]$ \\
\hline Median dextrose rate [IQR] (g/hr): & $5.2[3.4-6.5]$ & $5.2[3.3-6.5]$ & $5.2[3.3-6.5]$ & $5.2[3.4-6.5]$ \\
\hline
\end{tabular}


Table 5. Overall cohort summary statistics as reported by simulated 5 minute CGM from Monte Carlo simulations using each of the noise models (with alarm and 12.5 gram interventions).

\begin{tabular}{|l|c|c|c|c|}
\hline Summary Statistics (CGM) & Baseline (no noise) & Breton & Goldberg (full) & Goldberg (half) \\
\hline BG median $[\mathrm{IQR}](\mathrm{mmol} / \mathrm{L}):$ & 5.5 & $5.7[4.8-6.5]$ & $5.7[4.8-6.5]$ & $5.6[4.8-6.4]$ \\
\hline$\%$ BG within 4.4-6.1 mmol/L [IQR] & 53.9 & $47.9[47.8-48.1]$ & $47.1[46.9-47.4]$ & $51.9[51.7-52.1]$ \\
\hline$\% \mathrm{BG}<4.4 \mathrm{mmol} / \mathrm{L}[\mathrm{IQR}]$ & 15.5 & $16.3[16.1-16.4]$ & $17.1[17.0-17.1]$ & $15.4[15.3-15.5]$ \\
\hline$\% \mathrm{BG}<4 \mathrm{mmol} / \mathrm{L}[\mathrm{IQR}]$ & 8.7 & $9.2[9.0-9.4]$ & $9.7[9.6-9.8]$ & $8.2[8.1-8.3]$ \\
\hline$\% \mathrm{BG}<2.2 \mathrm{mmol} / \mathrm{L}[\mathrm{IQR}]$ & 0.28 & $0.23[0.22-0.25]$ & $0.15[0.14-0.16]$ & $0.09[0.09-0.10]$ \\
\hline Num patients $<2.2 \mathrm{mmol} / \mathrm{L}[\mathrm{IQR}]$ & 12 & $12[11-12]$ & $10[8-11]$ & $8[6-9]$ \\
\hline
\end{tabular}

results determined using the entire CGM sequence ( 5 minute values), representing what might be observed in the clinical setting if CGM monitoring was implemented. The baseline data is provided for comparison, and is the model-generated BG profile (based on 1-2hourly clinical data).

Table 2 shows that there are no clinically significant differences in the median BG levels between the baseline, Breton model, and Goldberg models. Time in the target 4.4$6.1 \mathrm{mmol} / \mathrm{L}$ band is also very similar between the baseline and CGM results and the differences are not considered clinically significant. Insulin and glucose administration remains unchanged with the addition of noise.

Table 3 shows the number of patients experiencing at least one hypoglycaemic event appears dependent upon the noise model. When using the Breton model the number of patients experiencing hypoglycaemia is unchanged from the baseline (12 events). However, with the Goldberg-full and half magnitude models, the number of hypoglycaemic events is reduced to as low as 8 per run.

The per-patient results in Table 4 are presented as a means of reducing the effect of very long stay patients on the overall results of the cohort. The results show similar trends to the overall cohort results and any differences seen are not clinically significant.

In Table 5 the BG median for each of the noise models is very similar to the results seen for the baseline (no noise). Time in the 4.4-6.1 mmol/L band has decreased $2-6 \%$ depending on the noise models and percent below $4.4 \mathrm{mmol} / \mathrm{L}$ along with percent below $4 \mathrm{mmol} / \mathrm{L}$ have increased slightly.
Interestingly, percent below $2.2 \mathrm{mmol} / \mathrm{L}$ has decreased for all 3 error models, suggesting the interventions are reducing the time spent in hypoglycaemia.

Figure 2 shows the BG profile for a patient who experienced severe hypoglycaemia. The top plot illustrates how a patient can become hypoglycaemic (739 hour) and remain there for approximately 1 hour, when not monitored by a CGM. The bottom plot shows the same patient being monitored by a CGM using the Breton noise model. Potential hypoglycaemia is detected prior to the 739 hour, and a 12.5 gram IV intervention is given, preventing a drop below $2.2 \mathrm{mmol} / \mathrm{L}$.

\section{DISCUSSION}

\subsection{Performance:}

The aims of this in-silico study were to show that nursing workload can be reduced, the level of glycemic control maintained, and early hypoglycemic detection/prevention implemented using CGM sensors in the ICU, despite potentially high levels of sensor noise in individual measurements. The performance of the CGM sensors in this role was evaluated in-silico using Monte Carlo simulations with stochastic CGM noise models and real ICU patient data.

Using CGMs to monitor BG levels in the ICU could improve patient safety by alerting nurses at the onset of potential hypoglycaemia. Early warning or detection of hypoglycaemia would allow it to be avoided or the length of the episode minimised.

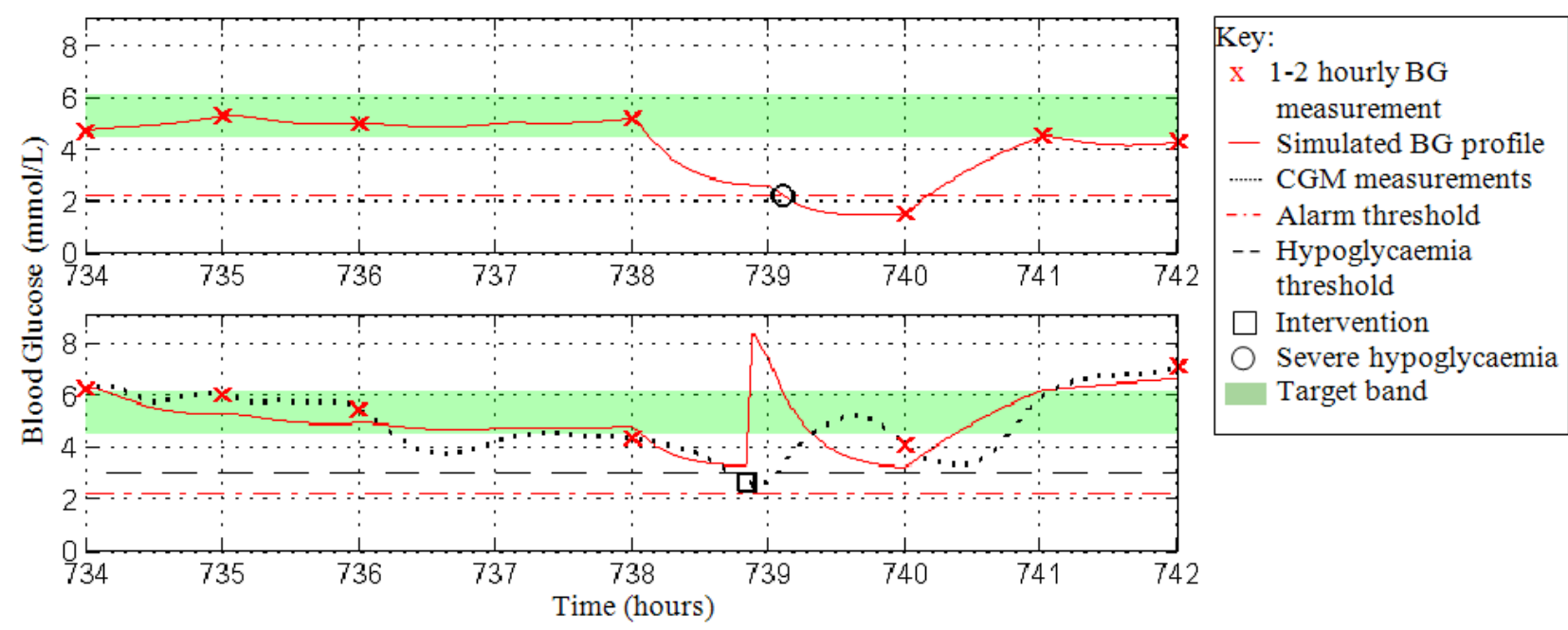

Figure 2. (Top) Blood glucose profile for a patient from the baseline simulation, showing a hypoglycaemic event at 739 hours. (Bottom) Blood glucose profile for the same patient when controlled by a CGM using the Breton noise model. A hypoglycaemic event is detected, so 12.5gram intravenous glucose is given just before 739 hours, preventing hypoglycaemia. 
The lack of clinically significant changes in the blood glucose control metrics shown in Table 2 suggest that the additional noise introduced by the CGM sensors has little effect, at least in the context of the SPRINT protocol. This may be because SPRINT is model-derived and both the inputs and outputs are discretised, so small sensor errors do not generally have a large effect on the protocol selected insulin and nutrition rates and thus the resulting blood glucose levels.

Table 3 shows that the median, mean and standard deviation BG levels are not clinically different between baseline and the modelled CGM results. The time in the 4.4-6.1 mmol/L band was reduced by $2-7 \%$ with the CGM results compared to the baseline, but this may be clinically acceptable given the potential advantages of CGMs in reducing the number and duration of hypoglycaemic episodes.

The number of patients experiencing severe hypoglycaemia did not increase from the baseline with the modelled CGM devices. The decrease in the number of hypoglycaemic events observed with the Goldberg models could be due to the independent nature of each error about the current actual BG level, which does not take into account any delay or timeseries behaviour. This allows the alarm/intervention algorithm to detect and avert hypoglycaemic events earlier than the Breton model. This is dependent on the type of error models used, and could likely be resolved with real, ICU specific, CGM data.

Table 3 shows that the total combined duration of severe hypoglycaemic events is reduced from 26 hours (9302 hours $\mathrm{x} 0.028 \%$ ) to between 9 and 21 hours (total for 10 Monte Carlo runs) depending on the noise model. With CGM data the number of patients experiencing hypoglycaemia may not be reduced however the reduction in duration of the events is still a clinically significant result given their negative impact on outcome (Egi et al., 2010)

A final significant result is the reflection on nursing time. SPRINT typically requires on average 16 measurements per day at 1.5 - 2 minutes per measurement (all tasks) (Lin et al., 2008b). Assuming a CGM was implemented with a noise model similar to that of Breton et al, the alarm/intervention algorithm used in this study would require on average 1 intervention per approximately 24 hours. With 4 calibration measurements per day and a single intervention, these results would reduce the total measurement burden $70 \%$ (11/day) or 17-22 minutes per patient per day, which is potentially clinically significant in a busy ICU environment.

\subsection{Limitations - Clinical:}

Clinical testing is required to confirm the results of this insilico study, even though the virtual patient simulation method used here is clinically well validated (Chase et al., 2007, Lonergan et al., 2006). The BG sequences used to drive SPRINT in this study were model-based, not real CGM output data. This analysis provides a strong initial proof of concept, however, testing and validation of the findings in a clinical setting is needed to confirm these results.

\subsection{Limitations - Signal Processing:}

Two separate stochastic noise models were used in this study providing 3 different CGM sensor noise profiles. The two Gaussian noise profiles are based on data from a study of CGMs in a medical ICU (Goldberg et al., 2004), however the model was created from reported sensor error statistics and therefore the time-series information about the errors is lost, hence they are assumed to be independent. The third noise profile was generated using the model reported in (Breton and Kovatchev, 2008), and while much more complete in terms of modelling glucose diffusion to the interstitium and the interdependence of errors, the model was derived for ambulatory type 1 diabetics and is therefore not necessarily the best model for ICU patients.

While not having a validated, ICU-specific CGM noise model, analysing the results of these 3 noise profiles shows little difference in the overall control (mean, median and standard deviation) of the patients and it is therefore anticipated that real CGM characteristics are captured somewhere between these three simulated models.

True sensor noise characteristics combined with more advanced filtering techniques would likely result in a much cleaner CGM data stream and hence a more reliable method for triggering alarms and interventions. The simple alarm algorithm may also have triggered false hypoglycemia alarms furthering the need for a more robust algorithm such as an integral-based method (Chase et al., 2006, Pretty et al., 2009). Thus, a better model would likely improve the performance outcomes and alarm data reported here. Hence, these results as presented are potentially conservative.

Calibration drift due to sensor degradation over time was not considered in this study. Without correction, calibration drift will show up as though the actual BG measurements were higher or lower in a relatively consistent manner as the sensor gain drifts (Kuure-Kinsey et al., 2006). This drift would cause the alarm to trigger early (possibly falsely) or late. However, such calibration drift is typically a function of the frequency and quality of calibration measurements, which can likely be controlled more readily in a critical care setting using sensors such as blood gas analysers. This issue also has not been quantified, to date, in the critical care setting with modern CGM devices.

\subsection{Summary:}

Finally, this paper is focused on proof of concept for the integration of CGMs into the ICU. The generally good results seen here with sub-optimal filtering and a conservative noise model serve primarily to show that the method is feasible and deserves further direct clinical investigation.

\section{CONCLUSION}

This paper has analysed in-silico the use of CGM sensors with simple filters and hypoglycaemia alarms to provide input to the SPRINT TGC protocol. CGMs, even with considerable noise, have no significant clinical impact on TGC under the SPRINT protocol. CGMs could potentially reduce the number and duration of hypoglycaemic events with suitable alarm and intervention algorithms. Finally, the use of CGMs with SPRINT could reduce nursing burden for 
measurement up to $70 \%$, potentially saving 17-22 minutes per patient, per day. All of these results justify clinical testing for validation, as well as highlighting the main issues in using CGMs for TGC in critical care.

\section{REFERENCES}

Braithwaite, S. S., Edkins, R., Macgregor, K. L., Sredzienski, E. S., Houston, M., Zarzaur, B., Rich, P. B., Benedetto, B. \& Rutherford, E. J. 2006. Performance of a dose-defining insulin infusion protocol among trauma service intensive care unit admissions. Diabetes Technol Ther, 8, 476-88.

Breton, M. \& Kovatchev, B. 2008. Analysis, Modeling, and Simulation of the Accuracy of Continuous Glucose Sensors. Journal of Diabetes Science and Technology, 2, 853-862.

Capes, S. E., Hunt, D., Malmberg, K. \& Gerstein, H. C. 2000. Stress hyperglycaemia and increased risk of death after myocardial infarction in patients with and without diabetes: a systematic overview. Lancet, 355, 773-778.

Chase, J., Andreassen, S., Jensen, K. \& Shaw, G. 2008a. The Impact of Human Factors on Clinical Protocol Performance - A proposed assessment framework and case examples. Journal of Diabetes Science and Technology (JoDST), 2, 409-416.

Chase, J., Lecompte, A., Shaw, G., Blakemore, A., Wong, J., Lin, J. \& Hann, C. 2008b. A Benchmark Data Set for Model-Based Glycemic Control in Critical Care. Journal of Diabetes Science and Technology (JoDST), 24, 584-594.

Chase, J. G., Hann, C. E., Jackson, M., Lin, J., Lotz, T., Wong, X. W. \& Shaw, G. M. 2006. Integral-based filtering of continuous glucose sensor measurements for glycaemic control in critical care. Comput Methods Programs Biomed, 82, 238-47.

Chase, J. G., Shaw, G., Le Compte, A., Lonergan, T., Willacy, M., Wong, X.-W., Lin, J., Lotz, T., Lee, D. \& Hann, C. 2008c. Implementation and evaluation of the SPRINT protocol for tight glycaemic control in critically ill patients: a clinical practice change. Critical Care, 12, R49.

Chase, J. G., Shaw, G. M., Lotz, T., Lecompte, A., Wong, J., Lin, J., Lonergan, T., Willacy, M. \& Hann, C. E. 2007. Model-based insulin and nutrition administration for tight glycaemic control in critical care. Curr Drug Deliv, 4, 283-96.

Clarke, W. L., Anderson, S., Farhy, L., Breton, M., GonderFrederick, L., Cox, D. \& Kovatchev, B. 2005. Evaluating the clinical accuracy of two continuous glucose sensors using continuous glucose-error grid analysis. Diabetes Care, 28, 2412-2417.

Egi, M., Bellomo, R., Stachowski, E., French, C. J., Hart, G. K., Taori, G., Hegarty, C. \& Bailey, M. 2010. Hypoglycemia and outcome in critically ill patients. Mayo Clin Proc, 85, 217-24.
Finney, S. J., Zekveld, C., Elia, A. \& Evans, T. W. 2003. Glucose control and mortality in critically ill patients. Jama, 290, 2041-2047.

Goldberg, P. A., Siegel, M. D., Russell, R. R., Sherwin, R. S., Halickman, J. I., Cooper, D. A., Dziura, J. D. \& Inzucchi, S. E. 2004. Experience with the continuous glucose monitoring system in a medical intensive care unit. Diabetes Technol Ther, 6, 33947.

Klonoff, D. C. 2005. A review of continuous glucose monitoring technology. Diabetes Technol Ther, 7, 770-5.

Krinsley, J. S. 2004. Effect of an intensive glucose management protocol on the mortality of critically ill adult patients. Mayo Clin Proc, 79, 992-1000.

Kuure-Kinsey, M., Palerm, C. C. \& Bequette, B. W. 2006. A dual-rate Kalman filter for continuous glucose monitoring. Conf Proc IEEE Eng Med Biol Soc, 1, 63-6.

Lin, J., Lee, D., Chase, J. G., Shaw, G. M., Le Compte, A., Lotz, T., Wong, J., Lonergan, T. \& Hann, C. E. 2008a. Stochastic modelling of insulin sensitivity and adaptive glycemic control for critical care. Comput Methods Programs Biomed, 89, 141-52.

Lin, J., Lee, D., Chase, J. G., Shaw, G. M., Le Compte, A., Lotz, T., Wong, J., Lonergan, T. \& Hann, C. E. 2008b. Stochastic modelling of insulin sensitivity and adaptive glycemic control for critical care. Computer Methods and Programs in Biomedicine, 89, 141-152.

Lonergan, T., Lecompte, A., Willacy, M., Chase, J. G., Shaw, G. M., Wong, X. W., Lotz, T., Lin, J. \& Hann, C. E. 2006. A simple insulin-nutrition protocol for tight glycemic control in critical illness: development and protocol comparison. Diabetes Technol Ther, 8, 191-206.

Mackenzie, I., Ingle, S., Zaidi, S. \& Buczaski, S. 2005. Tight glycaemic control: a survey of intensive care practice in large English hospitals. Intensive Care Med, 31, 1136.

Pretty, C., Chase, J., Lecompte, A., Shaw, G. \& Signal, M. 2009. Hypoglycemia Detection in Critical Care Using Continuous Glucose Monitors: An in Silico Proof of Concept Analysis. Journal of Diabetes Science and Technology, 4, 15-24.

Pretty, C. G., Chase, J. G., Le Compte, A., Shaw, G. M. \& Signal, M. 2010. Hypoglycemia Detection in Critical Care Using Continuous Glucose Monitors: An in silico proof of concept analysis. Journal of Diabetes Science and Technology, 4, 15-24.

Van Den Berghe, G., Wouters, P., Weekers, F., Verwaest, C., Bruyninckx, F., Schetz, M., Vlasselaers, D., Ferdinande, P., Lauwers, P. \& Bouillon, R. 2001. Intensive insulin therapy in the critically ill patients. N Engl J Med, 345, 1359-1367. 